\title{
A laboratory study of sediment deformation: stress heterogeneity and grain-size evolution
}

\author{
Neal R. Iverson, Thomas S. Hooyer and Roger LeB. Hooke \\ Department of Geology and Geophysics, University of Minnesota, Minneapolis, M.N 55455, U.S.A.
}

\begin{abstract}
In shearing sediment beneath glaciers, networks of grains may transiently support shear and normal stresses that are larger than spatial averages. Consistent with studies of fault-gouge genesis, we hypothesize that crushing of grains in such networks is responsible for surrounding larger grains with smaller grains. At sufficiently large strains, this should minimize stress heterogeneity, favor intergranular sliding and abrasion rather than crushing, and result in a self-similar grain-size distribution.

This hypothesis is tested with a ring-shear device that slowly shears a large annular sediment sample to high strains. Shearing and comminution of weak equigranular $(2.0-3.3 \mathrm{~mm})$ sediment resulted in a self-similar grain-size distribution with a fractal dimension that increased with shear strain toward a steady value of 2.85 . This value is significantly larger than that of gouges produced purely by crushing, 2.6, but it is comparable to values for tills thought to be deforming beneath modern glaciers, 2.8 to nearly 3.0. At low strains, under a steady mean normal stress of $84 \mathrm{kPa}$, variations in normal stress measured locally ranged in amplitude from 50 to $300 \mathrm{kPa}$ with wavelengths that were 100 times larger than the initial grain diameter. Crushing of grains, observed through the transparent walls of the device, apparently caused the failure of grain networks. At shearing displacements ranging from 0.7 to $1.0 \mathrm{~m}$, the amplitude of local stress fluctuations decreased abruptly. This change is attributed to fine sediment that distributed stresses more uniformly and caused grain networks to fail primarily by intergranular sliding rather than by crushing of grains. Sliding between grains apparently produced silt by abrasion and resulted in a fractal dimension that was higher than if there had been only crushing.

A size distribution with a fractal dimension greater than 2.6 is probably a necessary but not sufficient condition for determining whether a basal till has been highly deformed. Stress heterogeneity in subglacial sediment that is shearing through its full thickness should contribute to the erosion of underlying rock.
\end{abstract}

\section{INTRODUCTION}

Deformation of unlithified beds may be an important, if not dominant, mechanism of sediment transport by glaciers. For example, it has been suggested that the bulk of sediment deposited by certain lobes of the Laurentide ice sheet may have been transported as a water-saturated basal sediment layer undergoing pervasive simple shear (e.g. Alley, 1991). Continuous measurements of till deformation beneath modern glaciers are to date not sufficient to test this hypothesis. Such measurements indicate that the bed sometimes undergoes significant deformation, but that during periods of rising or high basal water pressure, motion is focused primarily at the glacier sole (Blake and others, 1994; Iverson and others, 1995). Nor are inferences from the geologic record sufficient. There are at present no unequivocal field criteria for determining whether tills deposited by former ice sheets have undergone the very high shear strains required to transport sediment long distances by this mechanism. The development of such criteria will require either extraordinary field control or appropriately configured laboratory experiments.

In this study we adopt the latter approach and focus on comminution processes and the evolution of grain-size during shearing of water-saturated sediment. Several basal tills that are thought to be deforming beneath modern glaciers have grain-size distributions that are selfsimilar with a characteristic fractal dimension of 2.8 to nearly 3.0 (Fischer, 1995; Hooke and Iverson, 1995; personal communication from S. Tulaczyk, 1995). Invoking theory developed for the similar problem of fault-gouge genesis (Sammis and others, 1987; Biegel and others, 1989), Hooke and Iverson (1995) suggested that such a grain-size distribution minimizes stress concentrations that crush grains. This should result in a steady fractal dimension at a sufficiently large shear strain. Here this hypothesis is tested with a rotary device that shears sediment to high strains while allowing continuous observation of the sample. The results clarify the relationship between the evolution of grain-size and the changing character of stress concentrations and comminution mechanisms with shear strain. 
FAILURE OF GRAIN BRIDGES AND COMMINUTION

Over sufficiently small length scales, stresses in a shearing granular material are heterogeneous. Experiments by Mandl and others (1977) showed that in some equigranular materials local stresses normal to the direction of shear deviated by as much as an order of magnitude from the mean normal stress applied to the sample. Load cells that measured the local normal stress had platen faces with diameters 30 times larger than the grain diameter, and thus, over smaller length scales, deviations from the mean normal stress may have been even larger.

Such observations suggest that grains may align transiently across the shear zone (Fig. la) and thereby support stresses normal and parallel to the direction of shear that may be much larger than spatial averages, a point demonstrated in experiments with shearing photoelastic discs (Drescher and de Josselin de Jong, 1972). Resultant alignments of grains support a coaxial compressive stress, $\sigma$, and have been called bridges (Mandl and others, 1977). They may fail either by fracture of a constituent grain or by frictional sliding and rolling of grains (Fig. 1a) (Biegel and others, 1989; Hooke and Iverson, 1995).

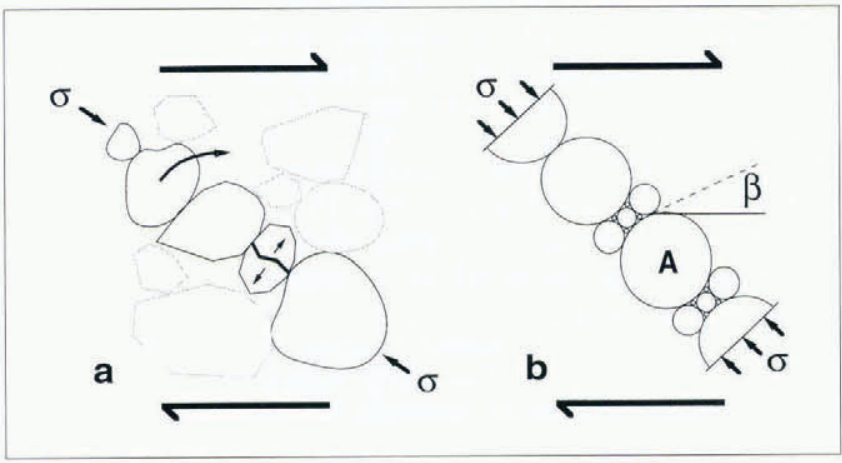

Fig. 1. (a) Segment of grain bridge that may fail by tensile fracture of a grain or by sliding between grains. (b) Idealized bridge in which grain $A$ is cushioned by smaller grains that distribule $\sigma$ over several contacts.

The partitioning between pervasive grain fracture (crushing) and sliding should depend primarily on the effective normal stress, the strength of the sediment grains, and the sediment grain-size distribution. To explore these dependencies, consider a simple two-dimensional bridge of grains like that considered by Sammis and others (1987) (Fig. 1b). The angle between the macroscopic shear plane and the intergrain slip plane is $\beta$. If $\sigma_{\mathrm{nl}}$ and $\sigma_{\mathrm{sl}}$ are the local stresses supported by the bridge normal and parallel to the macroscopic shear plane, respectively, and $\mu$ is a coefficient of friction that is defined parallel to $\beta$, then the bridge will fail by frictional sliding when

$$
\frac{\sigma_{\mathrm{sl}}}{\sigma_{\mathrm{nl}}} \geq \frac{\mu+\tan \beta}{1-\mu \tan \beta}
$$

(Biegel and others, 1989, equation 10). By substituting $\mu=\tan \omega$ and using a standard trigonometric identity, this inequality reduces to

$$
\sigma_{\mathrm{sl}} \geq \sigma_{\mathrm{nl}} \tan (\omega+\beta) .
$$

The sum, $\omega+\beta$, is equivalent to the friction angle of the familiar Coulomb failure criterion. A grain will undergo tensile fracture parallel to $\sigma$ if the compressive force exerted on it by an adjacent grain is sufficiently large. For simplicity we apply the relation used in the so-called Brazilian strength test (Jaeger and Cook, 1979, p. 169), which pertains strictly to compression of a cylinder across its diameter. Thus, for the two-dimensional case, fracture occurs when

$$
\sigma d_{\mathrm{N}} \geq \pi T d_{\mathrm{f}} / 2
$$

where $T$ is defined as the tensile stress required to split a grain in compression, $d_{\mathrm{f}}$ is the diameter of the grain that undergoes fracture, and $d_{\mathrm{N}}$ is the diameter of the largest neighboring grain. The product, $\sigma d_{\mathrm{N}}$, is the maximum force applied to the grain by a neighbor. Noting that $\sigma=$ $\left(\sigma_{\mathrm{nl}}^{2}+\sigma_{\mathrm{sl}}^{2}\right)^{\frac{1}{2}}$, combining this with Inequalities (2) and (3), and noting that $1+\tan ^{2}(\omega+\beta)=\sec (\omega+\beta)$, yields an inequality describing the conditions for fracture:

$$
\sigma_{\mathrm{nl}} \sec (\omega+\beta) d_{\mathrm{N}} \geq \pi T d_{\mathrm{f}} / 2 \text { for } 0<\omega+\beta<90^{\circ} .
$$

When this equality is not satisfied, bridges fail by frictional sliding or rolling of grains. Note that $\sec (\omega+\beta)$ increases monotonically over the applicable range of $\omega+\beta$, and thus the tendency for fracture of a given lithology increases with both $\sigma_{\mathrm{nl}}$ and $\beta$.

A reasonable expectation is that $\sigma_{\mathrm{nl}}$ scales with the mean effective normal stress on the sediment, defined as the difference between the ice-overburden pressure and the sediment pore-water pressure. Large effective normal stresses, therefore, should inhibit sliding by increasing friction between grains and by inhibiting the local dilation and consequent reduction in $\beta$ that are required to move grains past each other (Biegel and others, 1989). It is also obvious that for a given effective pressure and grain-size distribution, weaker lithologies will be more prone to fracture.

The grain-size distribution may affect the partitioning between fracture and sliding in two ways. As emphasized by Sammis and others (1987), the tendency for fracture of grains is increased if two particles of similar size are in contact. If several smaller particles surround a larger grain (Fig. 1b), $\sigma$ is distributed over several contacts, thus reducing $d_{N}$ in Inequalities (3) and (4) and the tendency for fracture. Sammis and others argue that this influence on fracture generally overrides the effect of the intrinsic particle strength. In addition, Biegel and others (1989) have suggested that if smaller grains surround larger grains over a wide range of length scales, there is a tendency to reduce the depth of interstices in which particles rest. They argue that this may reduce $\beta$, and thus, also reduce the likelihood of fracture.

In a fault gouge, in which fracture is the dominant mechanism of shearing owing to large effective normal stresses, Sammis and others (1987) argue that as shear strain increases, a grain-size distribution evolves in which the number of equal-sized nearest neighbors is minimized over a wide range of grain-sizes. This minimizes stress 
concentrations associated with bridging by distributing $\sigma$ over a maximum number of grain contacts and also perhaps by reducing the mean value of $\beta$. Thus, at high strains the likelihood of crushing decreases, and the potential for intergranular sliding is optimized. The resultant grain-size distribution is self-similar (fractal), such that if $N$ is the number of particles of diameter $d$, then

$$
N(d)=N_{0}\left(\frac{d}{d_{0}}\right)^{-m}
$$

where $N_{0}$ is the number of particles of reference size $d_{0}$, and $m$ is called the fractal dimension (e.g. Sammis and others, 1987). Plotting $\log N$ as a function of $\log d$ thus yields a straight line of slope $-m$. Geometric considerations discussed by Sammis and others (1987) suggest that $m$ should equal 2.58, consistent with natural gouges that had fractal dimensions within $5 \%$ of 2.60 . Experimentally simulated gouges also had a fractal dimension of 2.6 with a maximum variation of $12 \%$ (Biegel and others, 1989). In experiments, once this fractal dimension was reached at a sufficiently large shear strain, it remained steady with additional strain. These experiments also suggested that the steady value of 2.6 was independent of the initial grain-size distribution.

If crushing were the dominant mechanism of bridge failure in a shearing sediment layer beneath a glacier and if the layer had undergone sufficient shear strain, we might expect a grain-size distribution with a fractal dimension of 2.6. As noted earlier, however, samples from subglacial sediment layers that are thought to be deforming all have fractal dimensions between 2.8 and 3.0. Such values indicate an excess of fine particles. Hooke and Iverson (1995) suggested that there was also sliding between grains that generated excess fine particles by abrasion. Sliding between grains is more likely beneath a glacier, where effective normal stresses are generally lower than in fault zones.

\section{APPARATUS AND PROCEDURE}

To test these hypotheses and to study other mechanical properties of subglacial sediment, we have constructed a ring-shear device (Fig. 2a and b). The device shears a confined water-saturated sediment sample between parallel plates. The sample occupies an annular space that has an outside diameter of $0.6 \mathrm{~m}$ and a width of $0.115 \mathrm{~m}$. It is bounded at the top and bottom by permeable aluminum platens with teeth that grip the sample. Water in an internal reservoir that is open to the atmosphere moves into or out of the sample through the platens as the sample volume changes during shearing. The outer wall of the sample chamber is transparent hard-coated acrylic, so the outer margin of the sample can be observed continuously.

Shearing is accomplished by rotating the lower platen beneath the upper platen at a controlled rate with a variable-speed motor and gearboxes. The walls bounding the lower half of the sample move with the lower platen; the upper walls are fixed. The upper platen is screwed to a thick aluminum-and-steel plate, dubbed the normal-
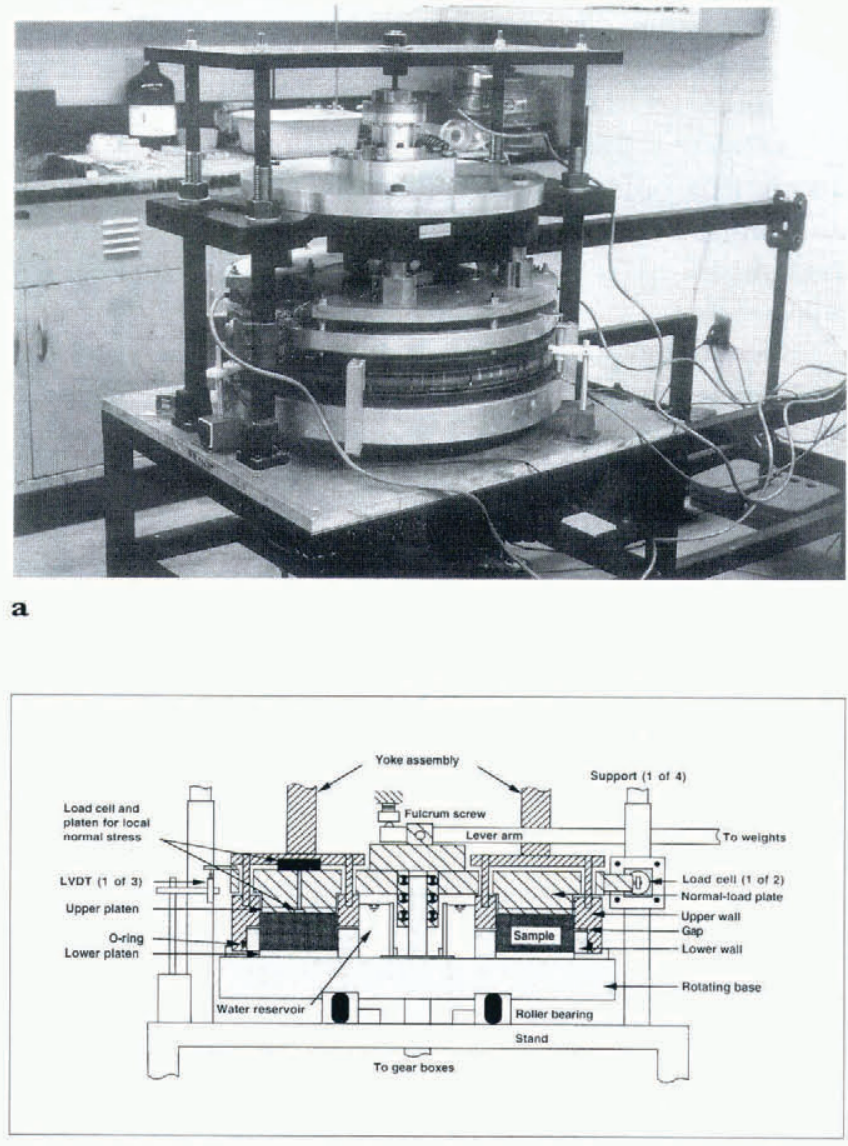

b

Fig. 2. (a) Photograph and (b) line drawing of the ringshear apparatus. Lightly shaded components rotate.

load plate, that is kept from rotating by two diametrically opposed roller platens that press against load cells (Fig. $2 b$ ). These load cells record the force on the normal-load platen that arises due to shearing. From this record, the shear strength of the sediment is calculated.

An assembly called the yoke (Fig. 2b) suspends the upper walls above the lower walls by a fraction of a millimeter. The yoke and upper walls are decoupled from the normal-load plate, so stresses on the walls are measured separately from stresses on the upper platen. Wall effects were of minimal concern in this study, although they will be discussed in detail in a separate paper aimed as assessing the suitability of the device for studies of till rheology.

A uniform normal stress is applied to the sediment by a lever arm with dead weights that presses down on the normal-load plate. The normal-load plate is free to move vertically as the sample thickness changes during shearing. The sample thickness is recorded by three displacement transducers that press on tabs that extend from the perimeter of the normal-load plate. Of special interest in this study is a platen, $18 \mathrm{~mm}$ in diameter, that is connected to a load cell and mounted flush with the surface of the upper platen (Fig. 2b). This system records local stresses normal to the shearing direction.

To achieve significant sediment comminution at rates of shearing and effective normal stresses appropriate for glaciers, we sheared a brittle but weak material: mudstone particles initially all $2.0-3.3 \mathrm{~mm}$ in diameter. The tensile strength of individual particles was estimated 
by measuring the force required to split grains in compression and calculating the tensile strength from this force (cf. Hiramatsu and Oka, 1966). Measured values were 1.0-4.0 $\mathrm{MPa}$. The mudstone, therefore, was about two orders of magnitude weaker than common crystalline rocks (see Jaeger and Cook, 1979, p. 146). Individual particles comprised silt- and clay-sized constituents.

The sediment was poured into the sample chamber and leveled to an initial uniform thickness ranging from 65 to $75 \mathrm{~mm}$. Linear passive markers were oriented vertically at several positions in the sample to record the distribution of shear strain. We have found that noodles (linguine) make excellent passive markers once hydrated. The sample was then loaded under a normal stress of $84 \mathrm{kPa}$ and saturated with water. In these experiments, the surface of the water reservoir was at atmospheric pressure. Although this may not be characteristic of unlithified glacier beds, where pore pressures are usually higher, we emphasize that it is the difference between the applied normal stress and the pore-water pressure, the effective normal stress, that should control intergranular stresses and comminution. An $84 \mathrm{kPa}$ stress is of the proper order for the effective normal stress beneath soft-bedded glaciers (e.g. Engelhardt and others, 1990 ).

After consolidation due to loading of the sediment had effectively stopped, shearing was initiated. The sample thickness, shear strength and local normal stress were recorded every minute. The outer margin of the sample was photographed periodically to record the deformation of strain markers and changes in grain-size, and to identify deformation mechanisms. The length of experiments was limited by the tendency for experimentally generated fine sediment to intrude into the gap between the upper and lower walls (Fig. 2b) at large shearing displacements $(1.0-2.5 \mathrm{~m})$.

During some experiments, shearing was stopped periodically, and the sediment was sampled. The grainsize distribution of the samples was analyzed by sieving the coarse fraction $(>63 \mu \mathrm{m})$ and using a laser counter (Lasentec) for the fine fraction (Agrawal and others, 1991, p. 119-128). We used the iterative method of Hooke and Iverson (1995) to calculate the number of grains and mean grain-size of each size class. This was necessary to compute the fractal dimension of the size distribution (Equation (5)). Some samples were collected intact and impregnated with epoxy (Card, 1994) for microfabric analyses.

Four experiments were conducted (MS-1-4) at a shearing rate of $880 \mathrm{~mm} \mathrm{~d}^{-1}$ and run to shearing displacements at the sample center ranging from 1.1 to $2.7 \mathrm{~m}$.

\section{RESULTS}

As expected, shear strain at the outer wall was focused near the plane of relative motion, which is marked by the gap between the upper and lower walls (Fig. 3a). Markers placed within the samples away from the walls indicated that the zone of deformation thickened rapidly toward the sample center, reaching a uniform thickness of 20-25 mm, 15-20 $\mathrm{mm}$ away from the walls. During the early stages of experiments, fracture of grains roughly parallel to the most compressive principal stress was commonly observed (Fig. 3b). As shearing progressed, a layer of fine sediment, $1-3 \mathrm{~mm}$ thick, became apparent near the gap where strain rates were highest. This layer thickened progressively before reaching a steady thickness of $10-15 \mathrm{~mm}$ after shearing displacements of $0.5-1.0 \mathrm{~m}$ (Fig. 3c). Away from the walls, this layer was $20-25 \mathrm{~mm}$ thick at the end of experiments.

Thin sections of impregnated sediment collected from the shear zone demonstrate that a wide range of grainsizes was produced by comminution associated with shearing (Fig. 4a). There was a distinct lack of similarsized grains in mutual contact, as is true for many basal tills (Fig. 4b) as well as fault gouge (Sammis and others, 1986).

Distributions of grain-size clearly demonstrate the production first of sand and then of a silt fraction at higher strains (Fig. 5a). Plotting $\log N$ as a function of $\log$ $d$ yielded a relatively linear relation with a slope of -2.37 after a shearing displacement of $0.15 \mathrm{~m}$ (shear strain $\sim 8$ ) (Fig. 5b), thus indicating that a self-similar size distribution had developed with a fractal dimension of 2.37 early in the experiments. Further shear strain and comminution increased the ratio of fine particles to coarse particles, thereby increasing the fractal dimension (Fig. 5b). The rate of increase was rapid at first, and then slowed down, apparently approaching a steady value of about 2.85 (Fig. 6a)

Local normal stresses fluctuated significantly, and the character of the fluctuations changed dramatically as the grain-size distribution approached a steady fractal dimension (Fig. 6b). Up to shearing displacements of $0.7-1.0 \mathrm{~m}$, fluctuations were large and of relatively consistent wavelength. Amplitudes ranged from about 50 to $300 \mathrm{kPa}$ with wavelengths of $100-200 \mathrm{~mm}$. These wavelengths were 5-10 times the diameter of the platen over which the measurements were made. At shearing displacements above approximately $1.0 \mathrm{~m}$, amplitudes and wavelengths became markedly smaller and less regular. In two of the experiments, MS-3 and MS-4, the time-averaged value of the local normal stress was larger than the applied mean stress by about $20 \mathrm{kPa}$. We attribute this difference to a small circumferential variation in sample thickness $(1.5 \mathrm{~mm})$ that resulted in slightly elevated normal stresses on thickened areas. The sediment shear strength tended to increase with strain, although this increase was small and not always monotonic (Fig. 6b).

At shearing displacements below about $40 \mathrm{~mm}$, the change in sample thickness was typical of many other loose granular materials (Fig. 6c). There was a small, rapid reduction in thickness of about $2-4 \%$, as shearing rearranged grains into a denser configuration, commensurate with the normal load (Lambe and Whitman, 1969 , p. 135). The thickness then briefly approached a steady value, before beginning a sustained decrease caused by comminution and the resultant filling of interstices with fine sediment. The rate of sample thinning due to comminution peaked at $100-200 \mathrm{~mm}$ of displacement and then gradually decreased. At the end of experiments, the sediment was still consolidating, although slowly. 

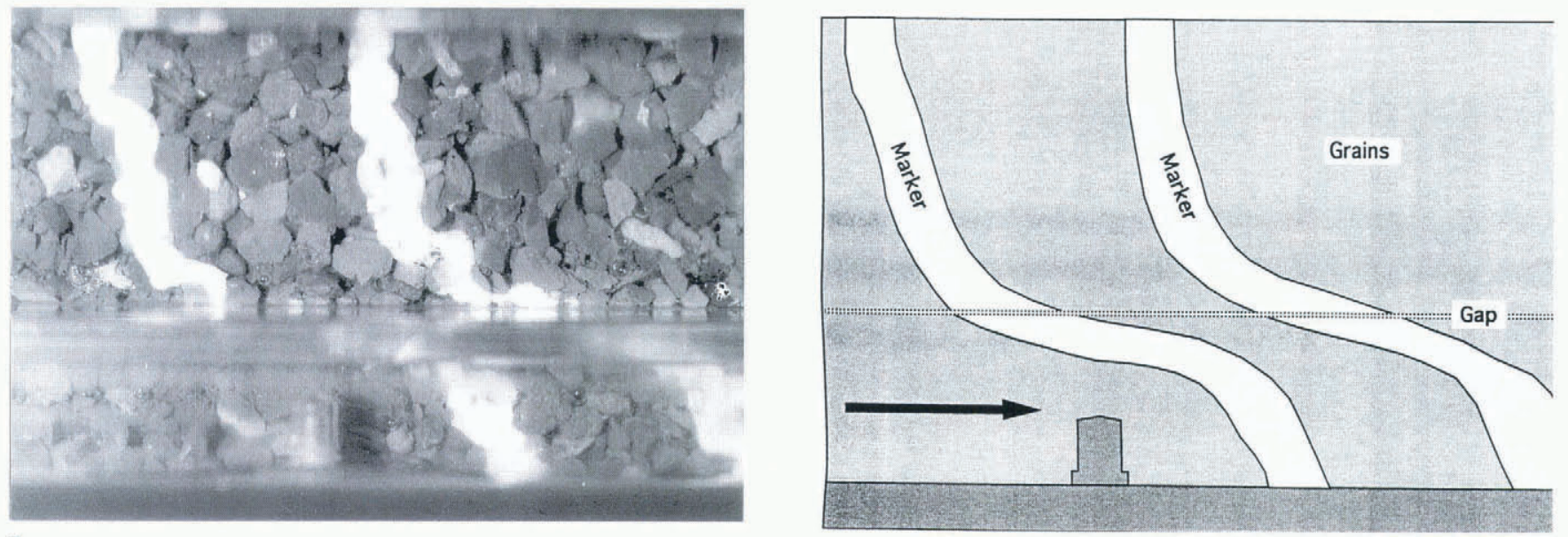

a
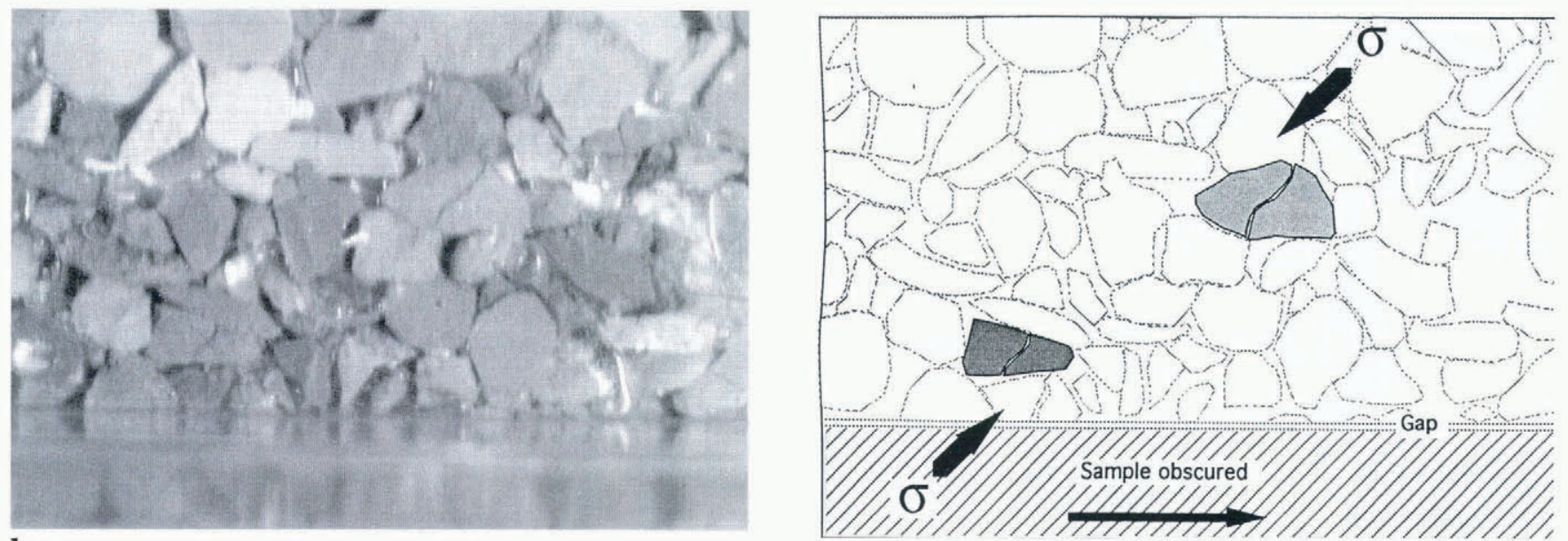

b
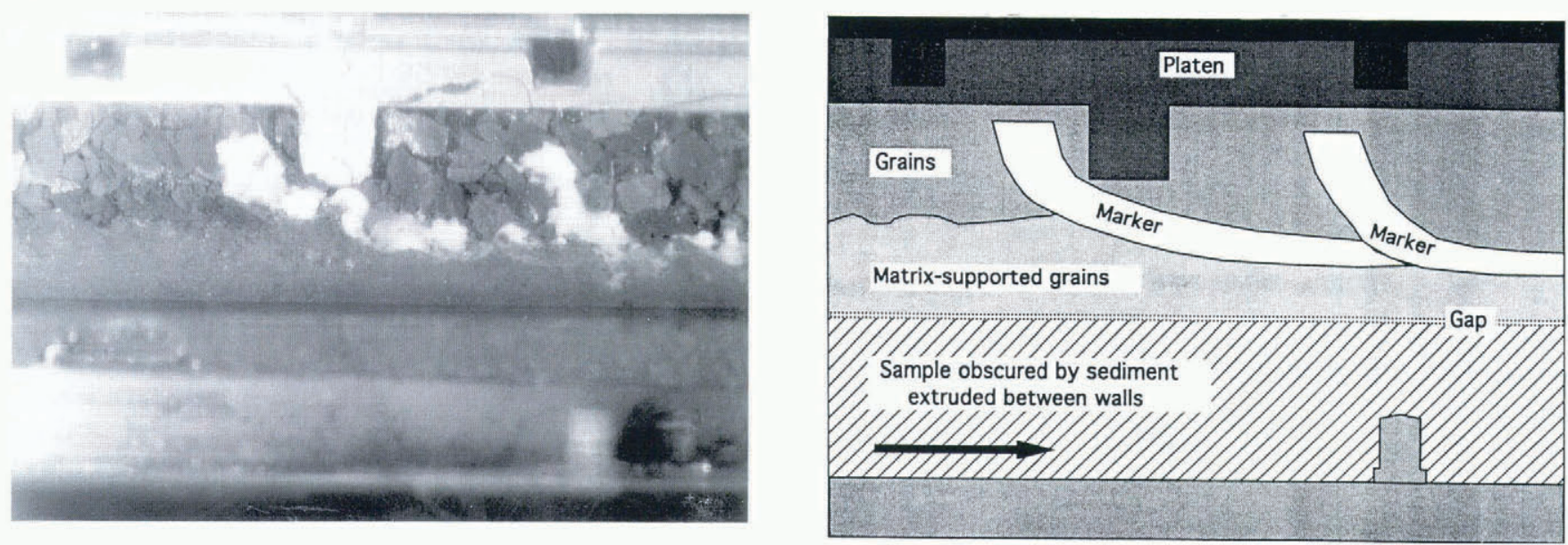

Fig. 3. Photographs taken through the outer wall of the apparatus and interpretive sketches of the photographs. The gap divides the rotating lower wall from the slationary upper wall. The screw that is visible in the lower wall in the top and bollom photographs and in the corresponding sketches is about $4 \mathrm{~mm}$ wide. (a) Strain markers after a shearing displacement of about $35 \mathrm{~mm}$. Visibility was poor immediately beneath the gap owing to an unpolished recess in the lower wall. (b) Tensile fracture of two grains roughly parallel to the most compressive principal stress. (c) Strain markers and fine sediment that resulted from comminulion afler a shearing displacement of $2.6 \mathrm{~m}$. The upper platen is visible due to thinning of the sample caused by comminution. Fine sediment that squeezed between the walls obscured the sample adjacent to the lower wall.

\section{DISCUSSION}

The local stress fluctuations in the first part of the experiments (Fig. 6b) coroborate the stress heterogeneity in shearing granular materials observed in other exper- iments (Drescher and de Josselin de Jong, 1972; Mandl and others, 1977). Stresses were concentrated over length scales of $100-200 \mathrm{~mm}$, which is broadly consistent with Mandl and others' data. Clearly the simple grain bridge of Figure la is an idealization; in reality such bridges 


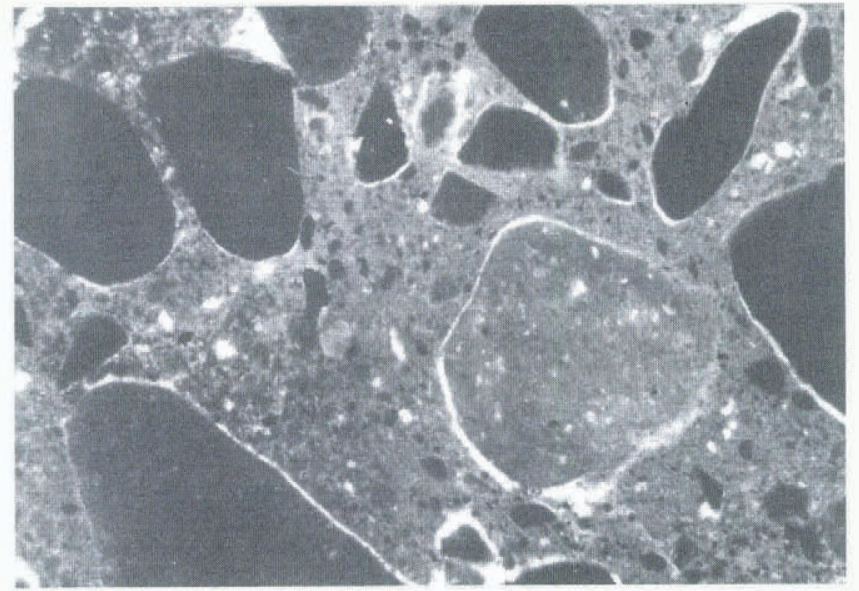

a

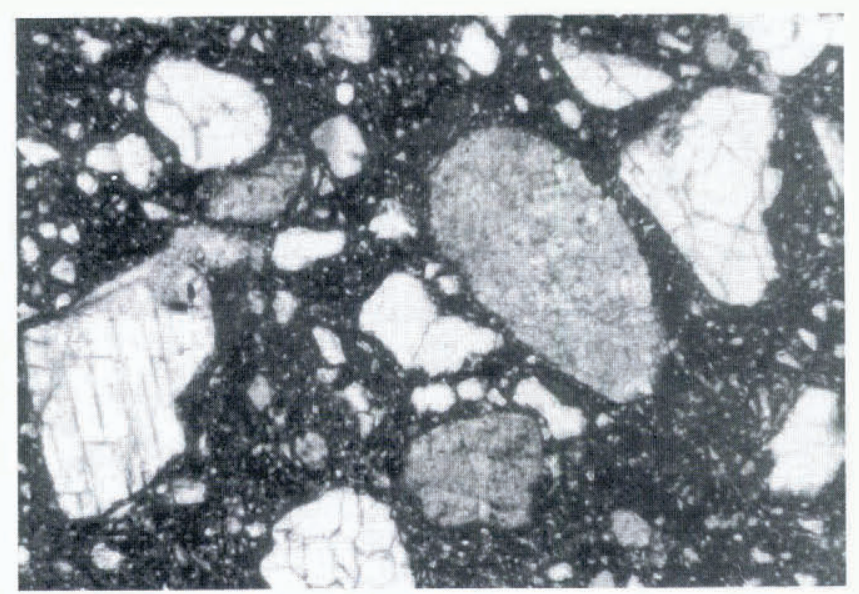

b

Fig. 4. (a) Thin section of sediment with a self-similar size distribution produced during an experiment. Note the lack of grains of similar size in mutual contact. The field of view is about $5 \mathrm{~mm}$. (b) Thin section of what is interpreted to be a basal till from the Des Moines lobe in southern Minnesota, U.S.A. The field of view is $2.5 \mathrm{~mm}$.

must combine with others to form networks that are probably complex geometrically but apparently have a characteristic length scale. Similar groups of more idealized grains that transiently behave as coherent units have been observed in both non-inertial (Drescher and de Josselin de Jong, 1972) and inertial (Drake, 1990) grain flows.

The change in the character of the stress fluctuations as the sediment shears and comminutes is best explained by a change in the partitioning between crushing and sliding of grains that comprise bridges. Initially neighboring grains were of similar size. As noted previously, this maximizes the potential for grain fracture by focusing the stress supported by a grain bridge over a minimum number of contacts (Inequality (4)). A reasonable conjecture, therefore, is that the initial large peaks in local normal stress reflect the stresses required to fracture grains in a bridge. This is supported by our observations of pervasive grain fracture early in the experiments (Fig. 3b). As grains are crushed and finer particles are produced, however, stresses are distributed over a greater number of contacts. Thus, at a sufficiently high ratio of fine to coarse grains, as indicated here by the fractal

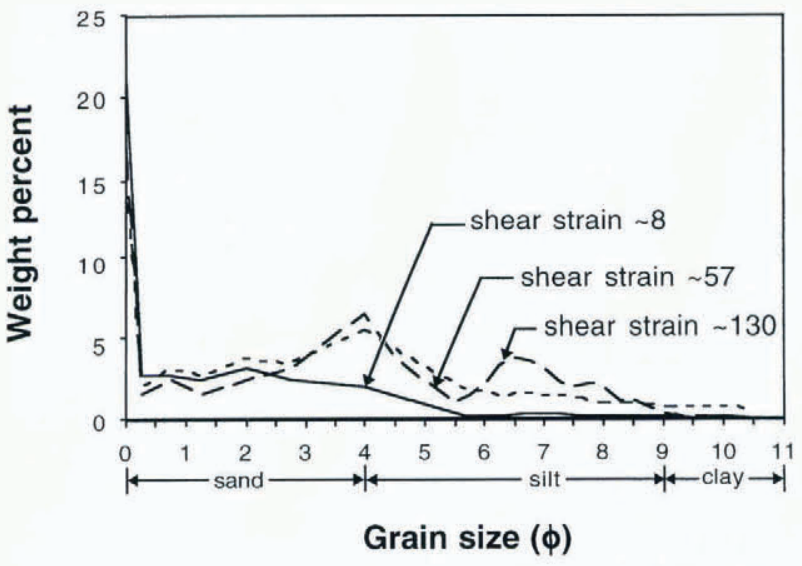

a

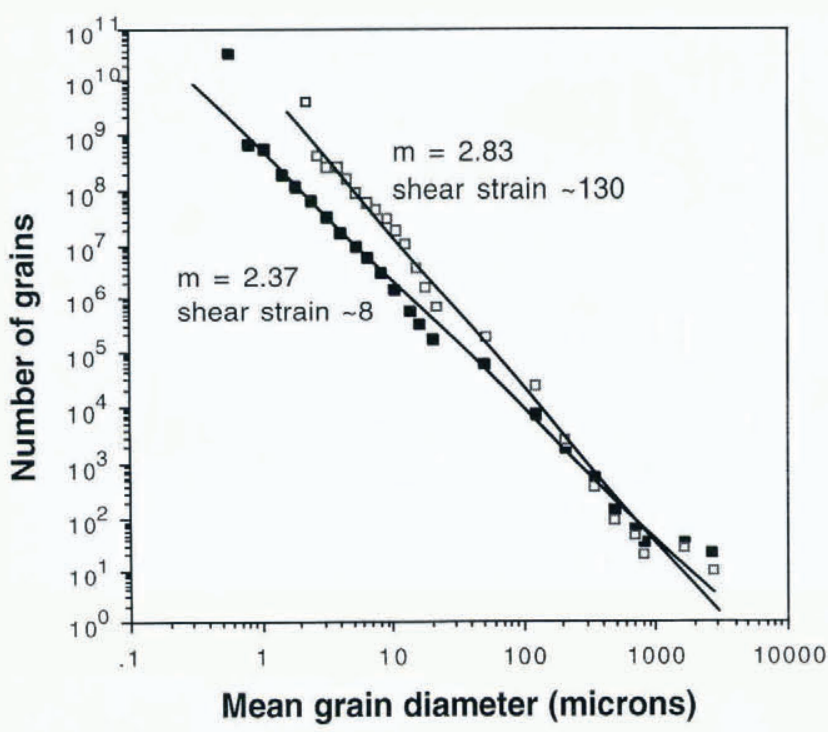

b

Fig. 5. (a) Histograms of the sediment grain-size distribution at shear strains of approximately 8, 57 and 130. (b) Plot of grain diameter vs number of grains at shear strains of 8 and 130 with best-fit lines. Samples were collected from a $10 \mathrm{~mm}$ thick zone immediately below the gap near the sample center.

dimension, Inequality (4) is satisfied less frequently. Bridges then fail primarily by sliding or rolling of grains past one another. The relatively abrupt change in the character of the stress fluctuations, rather than, for example, a steady reduction in the amplitude of the fluctuations, was unexpected, and suggests that there may be a relatively discrete grain-size distribution at which there is a change from one dominant mechanism of bridge failure to the other.

The grain-sizes produced by comminution confirm that crushing of grains dominated the failure of grain bridges early in the experiments (Fig. 5a). Sand was the principal grain-size produced at low strains, which is consistent with crushing of grains into several particles of comparable size. If sliding had dominated initially, a discrete silt-dominated fraction should have developed at low strains due to shipping of small particles from grain edges during abrasion (e.g. Haldorsen, 1981). Such a silt fraction did not develop until samples were highly strained. 


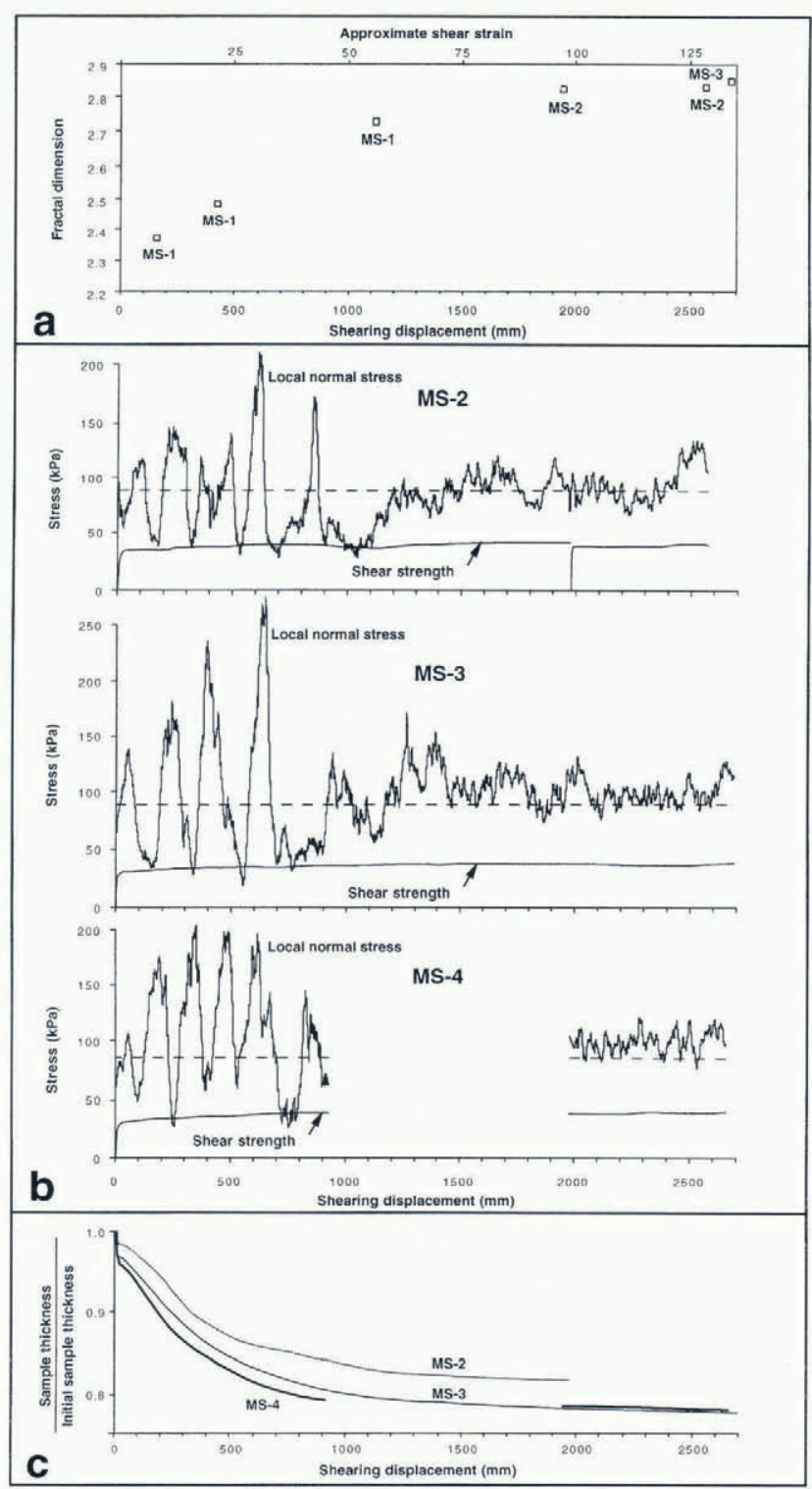

Fig. 6. (a) Fractal dimension of the sediment grain-size distribution as a function of shear strain and shearing displacement. The label on each point refers to the experiment in which the sample was taken. (b) Local normal stress and sediment shear strength during three experiments. The dashed line shows the mean normal stress on the sample. Data are not presented for MS-1 due to transient effects induced by frequent removal of the normalload plate for sampling. The gap in the MS-4 record resulted from a power failure. (c) Normalized sample thickness.

The measured rate of sample-thinning with shear strain and comminution also suggests a change in failure mechanism (Fig. 6c). Crushing of grains effectively collapses part of the grain skeleton, and thus should result in a greater rate of thinning than abrasion. Rates of sample thinning were high, although decreasing, during the first parts of the experiments when stress fluctuations were large and bridges were presumably failing more frequently by crushing (Fig. 6c). At larger shear strains, when stress fluctuations were smaller and bridges were presumably failing more frequently by sliding, rates of thinning were significantly lower and became nearly steady.
As noted previously, a fractal dimension of about 2.6 is expected if crushing is the only mechanism of bridge failure. The value of 2.85 that we observed reflects the excess of fines caused by abrasion. This value is similar to values for basal tills beneath modern glaciers. Note that as fine particles of a given size are produced by abrasion, they should be prone to crushing owing to their similar size. This may explain why fine particles remained fractally distributed, despite abrasion. Alternatively, the self-similar size distribution of the finest particles may reflect the sizes of particles that constituted the mudstone grains, although it is not obvious why the size distribution of such particles should be self-similar. A "terminal" grain-size is expected, below which further comminution becomes unlikely (e.g. Dreimanis and Vagners, 1971), and the size distribution is not self-similar. In these experiments, this grain-size was apparently less than several microns.

The shear strength of sediment increased slightly with shear strain and comminution. This does not support the hypothesis that $\beta$ in Inequality (4) is reduced as smaller grains resulting from comminution surround larger grains over a wide range of scales (Biegel and others, 1989). If $\beta$ had been steadily reduced, the friction angle of the sediment should have decreased steadily, resulting in a reduction in strength. We thus conclude that the change in the mechanism of bridge failure from crushing to sliding was caused primarily by distributing bridge stresses over an increasing number of contacts reduction of $d_{\mathrm{N}}$ in Inequality (4)), rather than by a reduction in $\beta$. The increase in shear strength recorded in our experiments may reflect the increase in bulk density caused by comminution (Fig. 6c). This is supported by the ring-shear experiments of Mandl and others (1977), who observed that as fine sediment gravitationally settled into the interstices at the base of a dilatent shear zone, the base of the shear zone strengthened.

\section{IMPLICATIONS}

These experiments help elucidate the processes by which sediment that is mobilized sufficiently beneath a glacier may be transformed into a till with a fractal grain-size distribution. During the early stages of shearing, networks of grains support shear and normal stresses that are much larger than spatial averages. In these experiments, the length scale of such networks was about 100 times the initial mean grain diameter. Because grains are of similar size initially, the stresses supported by such networks are focused on a small number of contacts, which optimizes the potential for crushing. Resultant finer grains distribute stresses over more contacts, thereby cushioning larger grains. A self-similar size distribution arises, therefore, in which smaller grains surround larger ones over a wide range of length scales. This size distribution homogenizes stresses by reducing significantly the strength and wavelength of grain networks. The homogenization of stresses due to comminution, together with the low effective pressure expected beneath glaciers with unlithified beds, favors failure by grain sliding rather than by crushing. This sliding causes abrasion of grains and the production of a fine fraction in excess of that produced 
purely by crushing. Thus, the steady fractal dimension of a subglacial sediment layer that has been highly deformed is larger than that of fault gouge in which crushing dominates.

One difference between these experiments and sediment deformation beneath glaciers is that the experimental sediment grains were significantly weaker than most till constituents. Thus, crushing was probably more rapid and extensive than in a natural till layer under the same effective pressure. Yet the angularity of grains of many sizes in natural deforming layers and the self-similar size distributions of such sediment (e.g. Hooke and Iverson, 1995) suggest that crushing is common, even for strong lithologies. Such crushing is probably caused by stress concentrations within individual grain networks that may greatly exceed those measured in these experiments, in which stresses were averaged over a platen area that was 50-80 times larger than the initial cross-sectional areas of grains. Thus, we believe that crushing and the evolution of grain-size were accelerated but not otherwise altered fundamentally by the weak sediment.

A second difference between the experiments and subglacial deformation is that shear zones in sediment beneath glaciers may be orders of magnitude thicker than those of the experiments. It is possible, although not obvious, that thicker shear zones may be less prone to the formation of grain networks for a given grain-size distribution, a problem that will be explored in future experiments on equigranular samples with contrasting grain-sizes. However, if strain tends to localize within subglacial sediment, as it does in non-inertial landslides (e.g. Skempton, 1985), for example, then natural and experimental shear-zone thicknesses may be similar.

The shear strain required to reach a steady fractal dimension may depend on a number of variables that were not studied here, including the effective pressure, lithology (tensile strength) of particles, initial grain-size distribution and perhaps strain rate. Despite these uncertainties, the fractal dimensions of basal tills that are thought to be deforming beneath modern glaciers are of similar magnitude and are significantly larger than 2.6. Thus, a reasonable conjecture, based on both the field and laboratory evidence, is that a till that either is not fractal or has a fractal dimension less than 2.6 has probably not undergone the extremely high shear strains required to account for most glacier motion. We emphasize, however, that a value greater than 2.6 in no way demonstrates that a till has undergone large strains. Grain comminution during transport in basal ice, incorporation of fine sediment from parent rock, or preglacial weathering may also give rise to a self-similar grain-size distribution with a comparable fractal dimension.

Stress heterogeneity in shearing sediment also has implications for glacial erosion. To help maintain continuity of the sediment that is thought to be deforming beneath Ice Stream B, Alley and others (1987) have suggested that a subglacial sediment layer may erode underlying rock, in agreement with some glacial geologic inferences (MacClintock and Dreimanis, 1964). Large spatial variations in normal stress, as measured in these experiments, will cause commensurate deviatoric stresses in the underlying rock. Such stresses should contribute to fracture and erosion of the rock, provided that subglacial sediment layers are sometimes mobilized through their full thickness. Because stresses may be concentrated over areas much larger than the cross-sectional areas of grains, cracks may grow in the rock over length scales significantly larger than those usually associated with abrasion.

These results also demonstrate that stress variations detected by instruments dragged through subglacial sediment may be caused by grain networks, in addition to temporal changes in effective pressure Iverson and others, 1994) and spatial variations in the density of clasts within a fine-grained matrix (Fischer and Clarke, 1994). For those attempting to extrapolate sediment behavior from point measurements beneath glaciers, however, it is encouraging that the grain-size distribution of subglacial till apparently minimizes mechanical heterogeneity. A continuum assumption, therefore, is perhaps more reasonable for till than for other sediment types that are better sorted.

\section{ACKNOWLEDGEMENTS}

The ring-shear device could not have been contructed without the generous and skillful assistance of J. Marchetti, foreman of the electrical engineering machine shop at the University of Minnesota. We thank S. Tulaczyk for his thorough review of the manuscript and the Minnesota Geological Survey for providing logistical support. We also thank the Limnological Research Center at the University of Minnesota for the use of their laser counter and R. W. Baker for helping with textural analyses. This work was supported by the U.S. National Science Foundation, OPP9224209.

\section{REFERENGES}

Agrawal, Y. C., I. N. Mc Cave and J. B. Riley, 1991. Laser diffraction size analysis. In Syvitski, J.P. M., ed. Principles, methods and application of particle size analysis. Cambridge, Cambridge University Press, 119-128.

Alley, R.B. 1991. Deforming-bed origin for southern Laurentide till sheets? J. Glaciol., $37(125), 67-76$.

Alley, R. B., D. D. Blankenship, C. R. Bentley and S. T. Rooney. 1987. Till beneath Ice Stream B. 3. Till deformation: evidence and implications. J. Geophys. Res., 92 B9), $8921-8929$.

Biegel. R. L.. C. G. Sammis and J. Dieterich. 1989. Frictional properties of simulated gouge having a fractal particle size distribution. $\mathcal{J}$. Struct. Geol., $11(7), 827-846$.

Blake, E. W., U.H. Fischer and G. K. C. Clarke. 1994. Direct measurement of sliding at the glacier bed. f. Glaciol., 40 136), 595-599.

Card, V.M. 1994. Ice-out, diatom community ecology, and varve formation in Big Watab Lake: phytoplankton response to interannual climate variation. Ph.D. thesis, University of Minnesota.

Drake, T. G. 1990. Structural features in granular flows. J. Geophys. Res., 95 B6), 86818696.

Dreimanis, A. and U.J. Vagners. 1971. Bimodal distribution of rock and mineral fragments in basal tills. In Goldthwait, R.P., ed. Till: a symposium. Columbus, OH, Ohio State University Press, 237-250.

Drescher, A. and G. de Josselin de Jong. 1972. Photoelastic verification of a mechanical model for flow of a granular material. f. Mech. Phys. Solids, 20. 337-351.

Engelhardt, H., N. Humphrey, B. Kamb and M. Fahnestock. 1990. Physical conditions at the base of a fast moving Antarctic ice stream. Science, 248 4951), 57-59.

Fischer, U.H. 1995. Mechanical conditions beneath a surge-type glacier. (Ph.D. thesis, University of British Columbia.

Fischer, U.H. and G. K.C. Clarke. 1994. Ploughing of subglacial sediment. 7. Glaciol., 40 134), 97-106. 
Haldorsen, S. 1981. Grain-size distribution of subglacial till and its relation to glacial crushing and abrasion. Boreas, 10 1), 91-105.

Hiramatsu, Y. and Y. Oka. 1966. Determination of the tensile strength of rock by a compression test of an irregular test piece. Int. J. Rock Mech. Min. Sci., 3, 89-99.

Hooke, R. LeB. and N. R. Iverson. 1995. Grain-size distribution in deforming subglacial tills: role of grain fracture. Geology, 23 1), 5760.

Iverson, N. R., P. Jansson and R. LeB. Hooke. 1994. In-situ measurement of the strength of deforming subglacial till. f. Glaciol., 40 (136), 497-503.

Iverson, N. R., B. Hanson, R. LeB. Hooke and P. Jansson. 1995. Flow mechanism of glaciers on soft beds. Science, 267 (5194), 80-81.

Jaeger, J.C. and N.G. W. Cook. 1979. Fundamentals of rock mechanics. Third edition. London, Chapman and Hall.
Lambe, T. W. and R. V. Whitman. 1969. Soil mechanics. New York, John Wiley and Sons,

MacClintock, P. and A. Dreimanis. 1964. Reorientation of till fabric by an overriding glacier in the St. Lawrence Valley. Am. J. Sci., 262 (1), $133-142$.

Mandl, G., L. N.J. de Jong and A. Maltha. 1977. Shear zones in granular material - an experimental study of their structure and mechanical genesis. Rock Mech., 9, 95-144.

Sammis, C., R. H. Osborne, J. L. Anderson, M. Banerdt and P. White. 1986. Self-similar cataclasis in the formation of fault gouge. Pure Appl. Geophys., $124(1), 53-78$.

Sammis, C., G. King and R. Biegel. 1987. The kinematics of gouge deformation. Pure Appl. Geophys., 125 (5), 777-812.

Skempton, A. W. 1985. Residual strength of clays in landslides, folded strata, and the laboratory. Geotechnique, 35, 3-18. 